\title{
Peter Weiden: «Viele psychosoziale Interventionen für Schizophrenie akzeptieren die fehlende Krankheits- einsicht der Patienten nicht»"
}

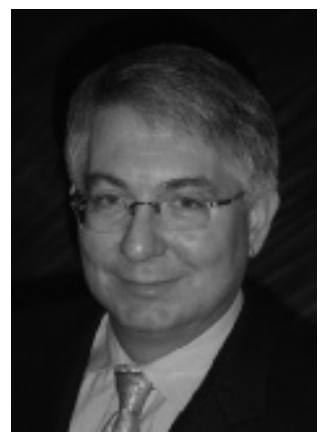

Peter J. Weiden, M.D., ist Professor für Psychiatrie und Direktor des Psychosenprogramms am Zentrum für Kognitive Medizin, Klinik für Psychiatrie, der University of Illinois at Chicago (UIC), USA. Ziel seiner Forschungen ist die Verbesserung der Behandlung schizophrener Patienten, indem er kognitiv-verhaltenstherapeutische Ansätze entwickelt, die die Zuverlässigkeit der Medikamenteneinnahme erhöhen sollen. Er ist außerdem bekannt durch seine Arbeiten zu psychoedukativen Angeboten für schizophrene Patienten und ihre Angehörigen. Das Gespräch führte Priv.-Doz. Dr. Rebekka Lencer, Oberärztin an der Klinik für Psychiatrie und Psychotherapie am Universitätsklinikum Schleswig-Holstein, Campus Lübeck, und Stipendiatin der Alexander von Humboldt-Stiftung am Zentrum für Kognitive Medizin, UIC, USA.

Herr Dr. Weiden, Sie sind ein weltweit anerkannter Experte auf dem Gebiet der Adhärenzforschung, der sogenannten «treatment adherence». Warum ist Forschung in diesem Bereich so wichtig für Patienten, die an Schizophrenie erkrankt sind?

Peter Weiden: Oh, vielen Dank für das Kompliment! Ich interessiere mich für dieses Gebiet schon seit meiner Facharztausbildung, lange vor Einführung der modernen Antipsychotika. Damals litten die meisten der mit Neuroleptika behandelten Patienten unter fürchterlichen extrapyramidalen Nebenwirkungen, sodass der Zusammenhang zwischen diesen höchst unangenehmen Nebenwirkungen und der hohen Rate von Therapieabbrechern auf der Hand lag. Allerdings musste ich bald feststellen, dass dieser offensichtliche Zusammenhang sehr viel komplizierter ist. Obwohl wir immer noch einen weiten Weg vor uns haben, bis wir dieses Problem wirklich gelöst haben werden, verstehen wir inzwischen besser, warum Patienten ihre Medikation oft selbständig absetzen. Das ist ein erster wichtiger Schritt dahin, effektivere Interventionen zu entwickeln.

Ist eine zuverlässige Medikamenteneinnahme, die «medication adherence», gleichzusetzen mit Therapieadhärenz?

Peter Weiden: Nein, sicher nicht. Nur auf die Medikamenteneinnahme zu fokussieren, ist zu eng gedacht. Dabei wird die viel weiter reichende Frage nach der therapeutischen Beziehung zwischen Arzt und Patient außer Acht gelassen. Eine unzuverlässige Medikamenteneinnahme ist gewiss ein nicht zu vernachlässigendes Problem, aber ich habe im Laufe meiner klinischen Tätigkeit auch gelernt, dass es nicht fair und oft ungerechtfertigt ist, einen Patienten als «non-adherent» zu bezeichnen, bevor die Wirksamkeit eines Medikamentes gesichert ist. Es sieht immer aus, als sei es viel einfacher, die Wirksamkeit eines Medikaments nachzuweisen als z.B. die Wirksamkeit eines Rehabilitationsprogramms, aber das stimmt nicht.

Non-Adhärenz ist kein auf die Schizophreniebehandlung beschränktes Problem, sondern in der gesamten Medizin weit verbreitet. Aber es gibt einen entscheidenden Unterschied im Vergleich zu chronischen körperlichen Erkrankungen. Wer Asthma oder Diabetes hat, mag zwar ein ComplianceProblem haben, aber meistens wird er doch einsehen, dass er krank ist. Im Falle der Schizophrenie wollen wir nun gerade die Patienten erreichen, die sagen: «Ich bin gar nicht krank, ich brauche keine Therapie.» Das heißt, wenn wir eine Intervention entwickeln, die auf Psychoedukation zu Schizophrenie aufbaut, erreichen wir diese Patienten damit gar nicht. Ich glaube, das Hauptproblem vieler psychosozialer Interventionen für Schizophrenie ist, dass sie die fehlende Krankheitseinsicht der Patienten nicht akzeptieren.

\section{KARGER}

Fax +497614520714

Information@Karger.de

www.karger.com (c) 2009 S. Karger GmbH, Freiburg

Accessible online at:

www.karger.com/ver
Priv.-Doz. Dr. Rebekka Lence

Klinik für Psychiatrie und Psychotherapie

Universitätsklinikum Schleswig-Holstein, Campus Lübeck

Ratzeburger Allee 160, 23538 Lübeck, Deutschland

Tel. +49 451 500-2444, Fax -4957

rebekka.lencer@psychiatrie.uk-sh.de 
Wo sehen Sie das Problem, wenn zuverlässige Medikamenteneinnahme als wichtigstes Therapieziel definiert wird?

Peter Weiden: Symptomkontrolle, soziales Funktionsniveau, Remission und Heilung - das sind alles Beispiele für klinische Therapieziele. Die zuverlässige Medikamenteneinnahme dagegen gehört nicht in diese Kategorie. Die zuverlässige Einnahme eines Antipsychotikums ist allerdings einer der wichtigsten Faktoren, die zu einer verbesserten Symptomkontrolle beitragen, und die Basis für andere psychosoziale Interventionen, die eine Verbesserung der Lebensqualität, des sozialen Funktionsniveaus und der Arbeitsfähigkeit bewirken und die Rückfallwahrscheinlichkeit senken. Für die klinische Arbeit impliziert dieses Verständnis, dass die Medikamenteneinnahme für den Patienten ein Mittel sein kann, seine Lebensziele zu erreichen. Aus der Sicht des Therapeuten sollte eine unzuverlässige Medikamenteneinnahme daher eher als ein Hindernis auf dem Weg zum gemeinsam mit dem Patienten definierten und von ihm gewünschten Therapieziel aufgefasst werden. So betrachtet wird Non-Adhärenz erst dann zum Problem, wenn sie negative Auswirkungen auf ein Therapieziel hat. Wird die Medikamenteneinnahme selbst zu einem Therapieziel gemacht oder dem Patienten als solches kommuniziert, wird die Bühne leider oft frei für eine ganze Reihe von Problemen in der Arzt-Patienten-Beziehung. So kommt es dann, dass Patienten den Eindruck haben, ihr Arzt sei mehr daran interessiert, dass sie seine Anweisungen befolgen, als daran, dass sie sich besser fühlen.

Ärzte neigen auch dazu, die Grenzen einer antipsychotischen Behandlung nicht klar genug darzustellen. Auch wenn dies mit der besten Absicht geschehen mag, um einen Patienten nicht auch noch zu ermuntern, seine Medikation abzusetzen, kann es dazu führen, dass Patient und Angehörige annehmen, die medikamentöse Behandlung führe zu einer vollständigen Heilung. So kann es dann vorkommen, dass Patienten residuale Symptome auf die fehlende Wirksamkeit des Medikaments zurückführen und es absetzen, obwohl es die Symptome bereits reduziert hat.

Eine zuverlässige Medikamenteneinnahme nicht als direktes Therapieziel aufzufassen, sondern als Mittel, um die vom Patienten definierten Therapieziele zu erreichen, kann dem Kliniker also helfen, die Einstellungen seines Patienten und dessen Verhalten im Zusammenhang mit seiner Erkrankung und Behandlung besser zu verstehen.

Sie haben auf den Unterschied zwischen Adhärenzverhalten und Adhärenzeinstellung aufmerksam gemacht. Worin besteht dieser Unterschied?

Peter Weiden: Normalerweise wird Adhärenz eher als Verhalten denn als Einstellung aufgefasst. Dass ein Patient seine Medikamente einnimmt oder nicht einnimmt, ist sicherlich ein Verhalten. Dagegen hängt es von seiner Einstellung ab, ob er die Medikamente nehmen will oder nicht. Dieser Unterschied wird häufig übersehen. Meiner Meinung nach stellt dieser Unterschied ein weiteres wichtiges Hindernis für die Entwicklung effektiverer Interventionen bei Adhärenzproblemen dar. Im Vergleich zum Adhärenzverhalten erfordern Probleme der Adhärenzeinstellung eine ganz andere Bewertung und ein anderes Management. Ein Patient, der seine Medikamente im Prinzip akzeptiert, aber zu desorganisiert ist, um zur Apotheke zu gehen, wäre ein gutes Beispiel für jemanden, der zwar eine positive Einstellung zu den Medikamenten hat, aber trotzdem nicht «adherent» ist. In diesem Fall ginge es darum, logistische Probleme zu lösen. Andererseits würde ein Patient, der seine Medikamente zwar nicht nehmen will, dessen Familie ihm diese aber ins Essen mischt, als «adherent» eingeschätzt, wenn man allein sein Verhalten beurteilen würde. Es gibt viele Patienten, bei denen sich Einstellung und Verhalten unterscheiden. Bei Patienten, die Vorbehalte gegenüber Medikamenten haben, muss es deshalb darum gehen zu verstehen, woher diese ablehnende Einstellung kommt, um eine Veränderung zu erreichen. Wer sich mit Adhärenzforschung beschäftigt, sollte sich entscheiden, ob er eine Einstellung oder ein Verhalten messen will und sein Studiendesign entsprechend ausrichten. Das wird leider oft vernachlässigt.

Wie groß schätzen Sie den Anteil der Patienten mit einer ablehnenden Einstellung im Vergleich zu Patienten mit einem unzureichenden Adhärenzverhalten?

Peter Weiden: Gute Frage, das hängt von sehr verschiedenen Faktoren ab. Generell denke ich: Je weniger wirksam ein Medikament, desto größer die Wahrscheinlichkeit, dass einer unzuverlässigen Medikamenteneinnahme ein logistisches Problem zugrunde liegt. Wenn aber ein Medikament theoretisch gut bei einem Patienten wirkt, er es aber trotzdem nicht regelmäßig einnimmt, ist es umso wahrscheinlicher, dass er das Medikament ablehnt.

Sie haben im Rahmen der großen Kohortenstudie PreFER (Prevent First Episode Relapse) am SUNY Downstate/Kings County Hospital Center in Brooklyn, New York, die Effektivität von Depotgaben zur Verbesserung einer verlässlichen Medikamenteneinnahme bei Ersterkrankten untersucht. Was zeigen Ihre Ergebnisse hinsichtlich Adhärenzverhalten und -einstellung?

Peter Weiden: Hinsichtlich des Verhaltens haben wir NonAdhärenz als einen Zeitraum von mindestens 14 Tagen innerhalb der ersten 12 Behandlungswochen definiert, in denen die Patienten die Medikamente nicht eingenommen hatten. In der Gesamtgruppe lag die Rate dafür bei immerhin $27 \%$. Im Einzelnen betrug die Rate in der Gruppe mit oraler Medikation $44 \%$ und war damit deutlich höher als in der Gruppe mit Depotmedikation, wo sie bei $11 \%$ lag.

Um nun Informationen über die Einstellung der Patienten zu bekommen, haben wir sie nach 12 Wochen gefragt, warum sie
Interview 
die Medikamente weiter genommen oder abgesetzt haben. Interessanterweise zeigte sich dabei zwischen den Patientengruppen mit oraler oder mit Depotmedikation kein Unterschied. Vielmehr war die Beziehung zum Therapeuten für die Patienten der Hauptgrund, die Medikamente weiterzunehmen; weder, dass sie dachten, sie bräuchten die Medikamente, noch dass sie sie absetzten, weil sie unter Nebenwirkungen litten. Außerdem bestätigte sich die Befürchtung vieler Therapeuten nicht, dass die Einstellung auf ein Depotpräparat gerade bei Ersterkrankten die therapeutische Beziehung negativ beeinflusst.

Was sind Ihrer Erfahrung nach die effektivsten Ansätze, die Medikamenteneinnahme zu verbessern?

Peter Weiden: Nach aktueller Studienlage ist es so, dass die Vermittlung von biomedizinischen Modellen der Schizophrenie und die Darstellung der Notwendigkeit der Medikamenteneinnahme, wie wir es aus patientenbezogenen Psychoedukationsprogrammen kennen, leider nicht zu einer Verbesserung der Medikamenteneinnahme führen. Dabei ist es mir wichtig klarzustellen, dass Psychoedukation für Angehörige sehr wirksam ist, um eine zuverlässige Medikamenteneinnahme bei den Patienten zu erreichen.

Als wir angefangen haben, ein kognitiv-verhaltenstherapeutisches Programm (KVT) zur Adhärenzverbesserung zu entwickeln, gingen wir von der Beobachtung aus, dass Patienten ihre Medikamente verlässlicher einnehmen, wenn man etwas findet, was ihnen dabei hilft, sich besser zu fühlen, und besonders, wenn die Patienten dies selber herausfinden. Das ist wirklich ein sehr starker Motivator. Und wir beobachteten einen weiteren Aspekt, nämlich ihre Beziehungen. Schizophrene Patienten sind was Beziehungen anbelangt nicht anders als die meisten von uns. Sie nehmen die Medikamente, wenn sie erkennen, wie wichtig sie dafür sind, ihre Beziehungen zu erhalten. Das zeigt, was für einen starken Einfluss drohende soziale Isolation auf die regelmäßige Medikamenteneinnahme haben kann.

Ich war also auf der Suche nach einer Intervention, die es erlaubt, das biomedizinische Modell «Ich habe Schizophrenie» zu umschiffen. Dabei stieß ich auf die KVT-Ansätze von Turkington und Kingdon aus Großbritannien, die es möglich machen, chronische Halluzinationen und persistierenden Wahn, aber auch Negativsymptome, zu reduzieren. Das Caveat dabei war allerdings, dass diese Interventionen immer zusätzlich zu einer Medikation angeboten wurden, denn sie sollen die Medikamenteneinnahme nicht ersetzen. Trotzdem dachte ich, aha!, wir können diesen Ansatz als Ausgangspunkt nehmen. Er beharrt nicht auf einem biomedizinischen Modell, sondern ist ein patientenzentrierter Ansatz, der damit beginnt, zusammen mit dem Patienten herauszufinden, was ihn am meisten belastet oder verletzt hat. Es geht darum, einen Behandlungsplan zu entwerfen, der auf die Punkte abzielt, die die Lebensqualität des Patienten seines Erachtens am meisten beeinträchtigen.
Welche Aspekte waren für Sie wichtig, als Sie Ihr KVTProgramm zur Verbesserung der Medikamenteneinnahme entwickelten?

Peter Weiden: Es ging besonders darum, die Basisprinzipien der KVT nicht aus den Augen zu verlieren: die Patientenzentrierung, die Orientierung auf ein Ziel, nicht auf dem biomedizinischen Modell zu beharren und immer konform mit der individuellen Agenda des Patienten zu bleiben. Wir haben mit dem von uns entwickelten Programm dann eine Pilotstudie gemacht, in der immerhin die Hälfte der Patienten, die KVT plus Psychoedukation erhalten hatten, auch noch nach 4 Monaten ihre Medikamente regelmäßig einnahmen. In der Kontrollgruppe, die nur Psychoedukation erhielt, hatten zu dieser Zeit alle Patienten die Medikation abgebrochen.

Theoretisch geht es also darum, dass wir Therapeuten dem Patienten unsere Meinung nicht aufdrängen und ihm ständig erzählen, warum die Medikamente für ihn gut sind. Stattdessen stehen die Pläne des Patienten im Mittelpunkt, und es ist wichtig, den Patienten, ganz im Sinne des geleiteten Entdeckens, herausfinden zu lassen, dass eine medikamentöse Stabilisierung ihm hilft, seine persönlichen Ziele zu erreichen.

Interessanterweise zeigte die Pilotstudie auch, dass die Patienten, die KVT erhalten hatten, sehr viel besser erklären konnten, warum sie Medikamente nehmen wollten oder auch nicht.

\section{Wie interpretieren Sie diesen Befund?}

Peter Weiden: Oh, er hat uns zunächst sehr gefreut. Wir gehen davon aus, dass Patienten, die ihre Abneigung und ihre Bedenken gegenüber Medikamenten formulieren können, im Lauf der Zeit eher in der Lage sind, die möglichen Vorteile einer medikamentösen Behandlung zu verstehen, als wenn die Medikamente ihnen aufgedrängt werden und sie sie dann ablehnen. Die Patienten, die KVT bekommen hatten, konnten auch besser beschreiben, dass Medikamente keine Wunder bewirken und manche Symptome trotz regelmäßiger Einnahme fortbestehen. Das halten wir für einen sehr gesunden Schritt.

Ist KVT ein Therapieansatz, mit dem wir grundsätzlich alle Patienten, die an einer Schizophrenie erkrankt sind, erreichen können?

Peter Weiden: Das ist eine sehr wichtige Frage, denn KVT kann nicht bei jedem helfen. Wenn ein Patient seine Medikamente nicht nimmt, weil sie nicht wirken oder weil er viele Nebenwirkungen bekommt oder weil er kognitiv zu unstrukturiert ist, dann wird auch KVT ihm nicht helfen können. Wir müssen sicherlich noch weiter daran arbeiten, maßgeschneiderte Adhärenz-Interventionen für spezifische Probleme einzelner Patienten zu entwickeln. 
Worin unterscheidet sich Ihr Ansatz vom Ansatz des «Shared Decision Making»?

Peter Weiden: Oh ja, dieser Ansatz ist jetzt auch in den USA sehr beliebt. Ich denke aber, dass er viel weniger auf die persönlichen Ziele des Patienten fokussiert; die Frage ist, wie der Therapeut damit umgeht, wenn die Ziele des Patienten sehr vom Standard klinischer Therapieziele abweichen. Dann ist es sicherlich sehr schwierig, den Prozess einer gemeinsamen Entscheidungsfindung zu initiieren.

Wie gut kommen klinisch tätige Therapeuten mit dem KVTAnsatz zurecht?

Peter Weiden: Das ist eine sehr interessante Frage. Alle Therapeuten, die in unserer Pilotstudie geschult wurden, waren sehr gute Kliniker und konnten sehr gut mit schwer kranken Patienten umgehen. Aber sie waren es auch gewohnt, die Patienten recht schnell zu belehren, was ihre Symptome waren und welche Medikamente sie brauchten. Den Klinikern fiel es sehr, sehr schwer, den Patienten zuzugestehen, aus ihrer Sicht zu schildern, was ihnen gut tut und was nicht, und zuzulassen, dass Patienten sagen, welche Medikamente oder Therapien ihnen helfen oder warum sie keine Medikamente wollen. Viele Therapeuten korrigieren die Patienten reflexartig, sobald sie etwas «Falsches» sagen, aber genau das beendet den Dialog.

\section{Wie können wir aus dieser Sackgasse herauskommen?}

Peter Weiden: Ich habe einmal aus Spaß gesagt, unser wichtigstes Werkzeug ist eine dicke Rolle Klebeband, die wir in die Ecke unseres Büros stellen. Immer wenn wir drauf und dran sind, den Patienten zu korrigieren, müssen wir uns vorstellen, unseren Mund zuzukleben, damit der Patient die Chance bekommt, uns mehr über seine Sicht der Dinge zu erzählen. Uns allen ging es so, dass wir am Ende der KVTInterventionen sehr viel über die Patienten gelernt hatten. Uns, und wir nehmen an, auch den Patienten, hat die gesamte Therapie sehr viel Spaß gemacht. Es war ein echter Dialog und eine echte Zusammenarbeit mit den Patienten, nicht so sehr eine Konfrontation Ich-gegen-Dich. Wir haben dafür den Namen «Health Dialogue Intervention» gefunden, um von dem Medikamentenmonolog wegzukommen, der sonst oftmals auf die Patienten einprasselt. In Zukunft möchten wir mit den Patienten noch weiter von der Medizin weg hin zu Fragen allgemeiner Gesundheit und Gesundheitsvorstellungen kommen. Wir glauben, dass das die Adhärenz deutlich verbessern würde. Ich sage glauben, weil es bisher nur eine Pilotstudie gibt und wir noch nicht genau wissen, ob dieser Ansatz wirklich dazu beitragen wird, unseren Patienten ein besseres Leben zu ermöglichen.

Da bin ich auch sehr gespannt und bedanke mich für das Interview.

You are welcome. 